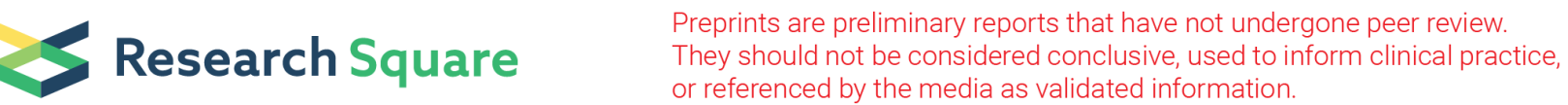

\section{Human Blastocyst is Susceptible to SARS-CoV-2 Infection based on Protein Level Examination of Key Entry Factors in Human Oocytes and Early Embryos}

xingping liu

Institute of Reproductive Health of Tongji Medical College of Huazhong University of Science and Technology https://orcid.org/0000-0001-9391-5717

Bing-xin Ma

Reproductive Medicine Center,Tongji Hospital, Tongji Medical College, Huazhong University of Science and Technology

Li-quan Zhou ( $\nabla$ zhouliquan@hust.edu.cn )

Huazhong University of Science and Technology https://orcid.org/0000-0002-9332-9408

Research

Keywords: SARS-CoV-2, Oocyte, Blastocyst, ACE2, TMPRSS2, NRP1

Posted Date: March 15th, 2021

DOl: https://doi.org/10.21203/rs.3.rs-288745/v1

License: (9) (1) This work is licensed under a Creative Commons Attribution 4.0 International License.

Read Full License 


\section{Abstract}

Objective

ACE2, TMPRSS2 and NRP1 are key factors for SARS-CoV-2 infection. Here, we used immunofluorescence to examine the expression patterns of ACE2, TMPRSS2 and NRP1 in human oocytes and different stages of preimplantation embryos to investigated the susceptibility to be infected by SARS-CoV-2.

Methods

We collected human GV oocytes and different stages of early embryos donated by patients and then performed immunofluorescence followed by confocal microscopy for signals of ACE2, TMPRSS2 and NRP1 proteins in these oocytes and embryos.

Results

We found that ACE2 was abundant in both inner cell mass and trophectoderm at blastocyst stage, while TMPRSS2 was mainly enriched in trophectoderm. Both of the two factors had faint signal in cleavage embryos and oocytes. In contrast, NRP1 was barely detectable in oocytes or any stage of early embryos.

\section{Conclusion}

Taken together, we propose that human blastocysts, instead of human oocytes and other stages of early embryos, are susceptible to be infected by SARS-CoV-2. Therefore, specific attention should be paid to manipulation of human blastocysts in assisted reproductive technology.

\section{Background}

Studies show that in developing countries where economies are underdeveloped, pandemics tend to bring baby booms. Attention to reproductive health issues during the COVID-19 pandemic is therefore extremely important. There are cases reporting positive results for SARS-CoV-2 in placenta and umbilical cord blood [1], and the presence of antibodies against SARS-CoV-2 in some newborns [2], indicating that there is possibility of infection before delivery by mother-to-child transmission. However, in two cases of women who tested positive for SARS-CoV-2, no viral RNA was detected in oocytes collected through ovarian stimulation [3]. Whether human oocytes and early embryos can be infected with SARS-CoV-2 remains elusive. SARS-CoV-2 was reported to enter cells through binding the cell membrane receptor ACE2, and then initiating entering through TMPRSS2 [4]. SARS-CoV-2 may be able to enter endometrial stromal cells through ACE2 receptor, and cause pathological manifestations in women with COVID-19, including increased risk of early pregnancy loss [5]. Studies have found co-expression of ACE2 and TMPRSS2 in primate ovarian cells. Moreover, ACE2 level was high in granular cells, but extremely low in TMPRSS2 level; ACE2 expression was low in endometrium, and TMPRSS2 expression was moderate [6] [7]. However, another study did not detect TMPRSS2 in oocytes by real-time PCR [3]. Single-cell transcriptome data analysis showed that ACE2 maintained a high level from the fertilized egg stage to the 4-cell embryo 
stage, and the expression was low from 8-cell embryo to morula stage. The expression levels of ACE2 in TE (trophectoderm) and ICM (inner cell mass) of blastocyst were close to that of fertilized eggs. On the contrary, TMPRSS2 has a continuous low expression from the fertilized egg to the morula stage, and the blastocyst has the strongest expression in TE [8]. Another SARS-CoV-2 host receptor neuropilin-1 (NRP1), which is known to bind furin-cleaved substrates could potentiate SARS-CoV-2 infectivity. NRP1 coexpression with ACE2 and TMPRSS2 markedly enhanced viral infection [9]. NRP1 is abundant in endothelial and epithelial cells of the respiratory tract [10], vascular endothelial cells and trophoblast cells in the villi [11], but the expression profile of NRP1 in oocytes and early embryos is not clear. Few experiments have confirmed the protein level of above factors potentially mediating SARS-CoV-2 infection in human oocytes and early embryos. Exploring the potential of the vertical transmission of SARS-CoV-2 infection through gametes and preimplantation embryos has significance of guiding ART (assisted reproductive technology) treatment and normal pregnancy during current coronavirus pandemic.

\section{Results}

To elucidate protein levels of factors potentially mediating SARS-CoV-2 infection, we collected human GV oocytes and different stages of early embryos donated by patients. Next, we performed immunofluorescence followed by confocal microscopy for signals of ACE2, TMPRSS2 and NRP1 proteins in these oocytes and embryos (Fig. 1 and Fig. 2).

Notably, ACE2 was present in both ICM and TE, while TMPRSS2 was mainly observed in TE. In contrast, both of the two factors had weak expression in cleavage embryos and GV oocytes. What's more, NRP1 is hardly detectable in oocytes and any stage of embryos. To verify the effectiveness of NRP1 antibody, we also performed immunofluorescence for signals of NRP1 proteins in embryo stem cells (Fig. S1).

We also analyzed the transcriptome data for human ACE2, TMPRSS2 and NRP1 genes from public database (Fig. 3). Generally, ACE2 transcript maintains a relatively high level in oocytes and from the fertilized egg to the four-cell stage embryo, but its level becomes extremely low from 8-cell embryo to morula stage. On the contrary, TMPRSS2 has a continuously low expression level in oocytes and from the fertilized egg to the morula stage. It should be noted that both ACE2 and TMPRSS2 transcripts are enriched in TE of blastocyst, and this is in agreement with our immunofluorescence result. Moreover, similar like its protein level, NRP1 transcript is not expressed in oocytes or early embryos.

\section{Discussion}

Our work focuses on documenting the protein level and localization of the three factors ACE2, TMPRSS2, NRP1 in human GV oocytes and early embryos, so as to predict the potential of SARS-CoV-2 infection. This work provides important information on how to safely perform assisted reproductive technology during the epidemic, and provide reference for asymptomatic infected and mild patients to choose the timing of pregnancy. According to the expression and distribution of ACE2, TMPRSS2 and NRP1, oocytes 
and cleavage stage embryos seem to have very low risk to be infected by coronavirus, and may be more suitable for embryo transfer during the epidemic. Despite of this, considering that viruses may infect cells through other receptors like HDL-scavenger receptor B type 1 [12], analyzing the protein expression of other receptors will be helpful to further explore the exact risk of SARS-CoV-2 infecting oocytes and early embryos and its impact on assisted reproduction. Our shortcomings were that the types of collected samples and tested receptors were limited. It will be also informative to test oocytes or embryos from patients who are positive for SARS-CoV-2. Taken together, exploring the potential of vertical transmission of SARS-CoV-2 infection through gametes and preimplantation embryos has guiding significance for the safe provision of ART treatment.

\section{Conclusions}

Taken together, both immunofluorescence result and transcriptome analysis support that TE of human blastocyst is susceptible to SARS-CoV-2 infection (Table 1).

Table 1

Comparison of RNA and protein levels of cell receptors in different developmental stages. Note that RNA and protein levels are not always equal.

\begin{tabular}{|llll|}
\hline & ACE2 & TMPRSS2 & NRP1 \\
& RNA/Protein & RNA/Protein & RNA/Protein \\
\hline GV oocyte & $+/-$ & $-/-$ & $-/-$ \\
\hline Cleavage embryo & $+++/+$ & $-/-$ & $-/-$ \\
\hline TE & $+++/+++$ & $+++/++$ & $-/-$ \\
\hline ICM & $-/-$ & $++/-$ & $-/-$ \\
\hline
\end{tabular}

\section{Materials And Methods}

\section{Collection of human oocytes and preimplantation embryos}

Human GV oocytes from donor and preimplantation embryos by in vitro fertilization abandoned by patients were obtained from Reproductive Medicine Center of Tongji Hospital, Tongji Medical College, Huazhong University of Science and Technology (HUST). Ethical approval for the study was obtained by the CEIC (Ethics Committee for Clinical Research) of Tongji Medical College, HUST. Informed consent was obtained from all patients in this study.

\section{Immunofluorescence}


Oocytes and embryos were fixed with $4 \%$ paraformaldehyde for 20 min. After fixation, samples were washed 3 times in PBS/PVP (0.3\% PVP in PBS), and permeabilized with $0.2 \%$ Triton-100 for 20 min at RT (room temperature). Next, samples were blocked in $2 \%$ BSA for 1 hour, incubated with the primary antibodies ACE2 (1:200 dilution, abclonal, A12737), TMPRSS2(1:200 dilution, ab109131), NRP1(1:200 dilution, proteintech, $2 \mathrm{H} 3 \mathrm{~F} 6$ ) at $4^{\circ} \mathrm{C}$ overnight. Cell were then washed for 3 times in PBS/PVP, and incubated for $2 \mathrm{~h}$ at RT with corresponding secondary antibodies: Alexa Fluor 488 secondary antibody and Alexa Fluor 594 secondary antibody (Thermo Fisher Scientific). Nuclei were stained with 1X Hoechst 33342 (C1028). Confocal imaging was performed using laser-scanning microscope (LSM780, Zeiss) in Tongji Medical College. Fluorescence intensity profiles were analyzed using Zeiss LSM software (ZEN BLACK).

\section{RNA-seq Data}

The RNA-seq result of human oocyte and pre-implantation embryo was downloaded from a previously published database (GSE36552) [13].

\section{Declarations}

\section{Ethics approval and consent to participate}

Ethical approval for the study was obtained by the CEIC (Ethics Committee for Clinical Research) of Tongji Medical College, HUST. The committee's reference number is $(S 314,2020)$. Informed consent was obtained from all patients in this study.

\section{Consent for publication}

Not applicable.

\section{Availability of data and materials}

The datasets used and/or analyzed during the current study are available from the publicly available GEO database (GSE36552).

\section{Competing interests}

The authors declare that they have no competing interests.

\section{Funding}


This work was supported by National Key R\&D Program of China [2018YFC1004001, 2018YFC1004502], the National Natural Science Foundation of China [NSFC 31771661], and the Fundamental Research Funds for the Central Universities [2019kfyXKJC074].

\section{Authors' contribution}

Li-quan Zhou designed the project. Xing-ping Liu and Bing-xin Ma performed the experiment and wrote the initial manuscript. Li-quan Zhou revised the manuscript. Xing-ping Liu and Bing-xin Ma contributed equally to this work. All authors have read and approved the final manuscript.

\section{Acknowledgements}

Not applicable.

\section{Authors' information}

Xing-ping Liu, Bing-xin Ma, Li-quan Zhou

Affiliations

Institute of Reproductive Health, Tongji Medical College, Huazhong University of Science and Technology, Wuhan, China

Xing-ping Liu

Reproductive Medicine Center, Tongji Hospital, Tongji Medical College, Huazhong University of Science and Technology, Wuhan 430030, China

Bing-xin Ma

Institute of Reproductive Health, Tongji Medical College, Huazhong University of Science and Technology, Wuhan, China

Li-quan Zhou

\section{References}

1. Pettirosso E, Giles M, Cole S, Rees M. COVID-19 and pregnancy: A review of clinical characteristics, obstetric outcomes and vertical transmission. Aust N Z J Obstet Gynaecol. 2020;60:640-59.

2. Gao J, Li W, Hu X, Wei Y, Wu J, Luo X, Chen S, Chen L. Disappearance of SARS-CoV-2 Antibodies in Infants Born to Women with COVID-19, Wuhan, China, Emerging infectious diseases, 26 (2020) 
2491-2494.

3. Barragan M, Guillén JJ, Martin-Palomino N, Rodriguez A, Vassena R, Undetectable viral RNA in oocytes from SARS-CoV-2 positive women, Human reproduction (Oxford, England), (2020).

4. Hoffmann M, Kleine-Weber H, Schroeder S, Krüger N, Herrler T, Erichsen S, Schiergens TS, Herrler G, Wu NH, Nitsche A, Müller MA, Drosten C, Pöhlmann S, SARS-CoV-2 Cell Entry Depends on ACE2 and TMPRSS2 and Is Blocked by a Clinically Proven Protease Inhibitor, Cell, 181 (2020) 271-280.e278.

5. Chadchan SB, Popli P, Maurya VK, Kommagani R, The SARS-CoV-2 receptor, Angiotensin converting enzyme 2 (ACE2) is required for human endometrial stromal cell decidualization, Biology of reproduction, (2020).

6. Stanley KE, Thomas E, Leaver M, Wells D. Coronavirus disease-19 and fertility: viral host entry protein expression in male and female reproductive tissues. Fertility sterility. 2020;114:33-43.

7. Henarejos-Castillo I, Sebastian-Leon P, Devesa-Peiro A, Pellicer A, Diaz-Gimeno P. SARS-CoV-2 infection risk assessment in the endometrium: viral infection-related gene expression across the menstrual cycle. Fertility sterility. 2020;114:223-32.

8. Chen W, Yuan P, Yang M, Yan Z, Kong S, Yan J, Liu X, Chen Y, Qiao J, Yan L. SARS-CoV-2 Entry Factors: ACE2 and TMPRSS2 Are Expressed in Peri-Implantation Embryos and the Maternal-Fetal Interface, Engineering (Beijing, China), 6 (2020) 1162-1169.

9. Cantuti-Castelvetri L, Ojha R, Pedro LD, Djannatian M, Franz J, Kuivanen S, van der Meer F, Kallio K, Kaya T, Anastasina M, Smura T, Levanov L, Szirovicza L, Tobi A, Kallio-Kokko H, Österlund P, Joensuu M, Meunier FA, Butcher SJ, Winkler MS, Mollenhauer B, Helenius A, Gokce O, Teesalu T, Hepojoki J, Vapalahti O, Stadelmann C, Balistreri G, Simons M, Neuropilin-1 facilitates SARS-CoV-2 cell entry and infectivity, Science (New York, N.Y.), 370 (2020) 856-860.

10. Daly JL, Simonetti B, Klein K, Chen KE, Williamson MK, Antón-Plágaro C, Shoemark DK, Simón-Gracia L, Bauer M, Hollandi R, Greber UF, Horvath P, Sessions RB, Helenius A, Hiscox JA, Teesalu T, Matthews DA, Davidson AD, Collins BM, Cullen PJ, Yamauchi Y, Neuropilin-1 is a host factor for SARS-CoV-2 infection, Science (New York, N.Y.), 370 (2020) 861-865.

11. Xu X, Yang XY, He BW, Yang WJ, Cheng WW. Placental NRP1 and VEGF expression in pre-eclamptic women and in a homocysteine-treated mouse model of pre-eclampsia. Eur J Obstet Gynecol Reprod Biol. 2016;196:69-75.

12. Wei C, Wan L, Yan Q, Wang X, Zhang J, Yang X, Zhang Y, Fan C, Li D, Deng Y, Sun J, Gong J, Yang X, Wang Y, Wang X, Li J, Yang H, Li H, Zhang Z, Wang R, Du P, Zong Y, Yin F, Zhang W, Wang N, Peng Y, Lin H, Feng J, Qin C, Chen W, Gao Q, Zhang R, Cao Y, Zhong H. HDL-scavenger receptor B type 1 facilitates SARS-CoV-2 entry. Nature metabolism. 2020;2:1391-400.

13. Yan L, Yang M, Guo H, Yang L, Wu J, Li R, Liu P, Lian Y, Zheng X, Yan J, Huang J, Li M, Wu X, Wen L, Lao K, Li R, Qiao J, Tang F. Single-cell RNA-Seq profiling of human preimplantation embryos and embryonic stem cells. Nat Struct Mol Biol. 2013;20:1131-9.

\section{Figures}




\section{Hoechst}

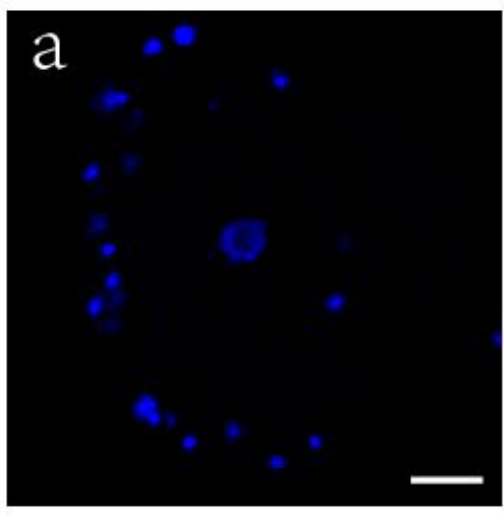

\section{d}

\section{Cleavage} embryo

\section{ACE2}

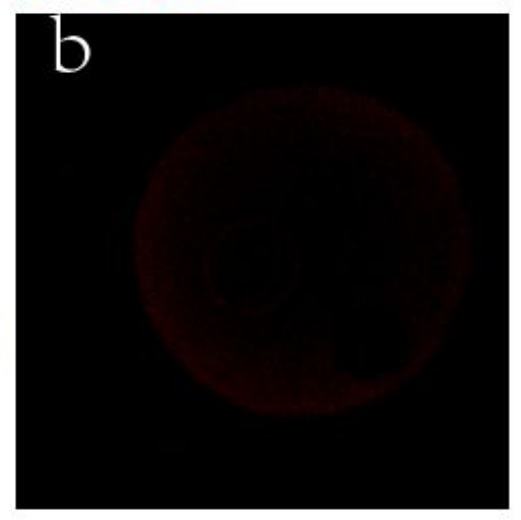

e

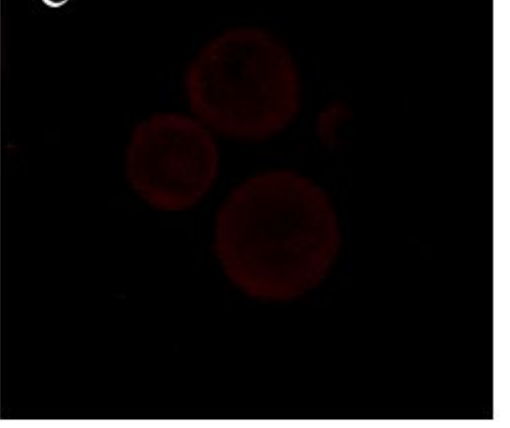

h

\section{NRP1}
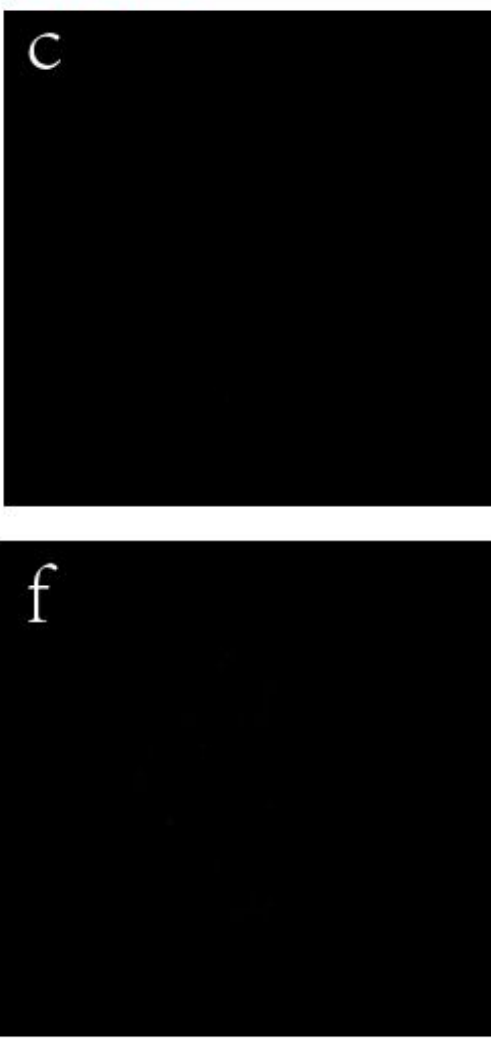

\section{i}

\section{Blastocyst}

\section{g}
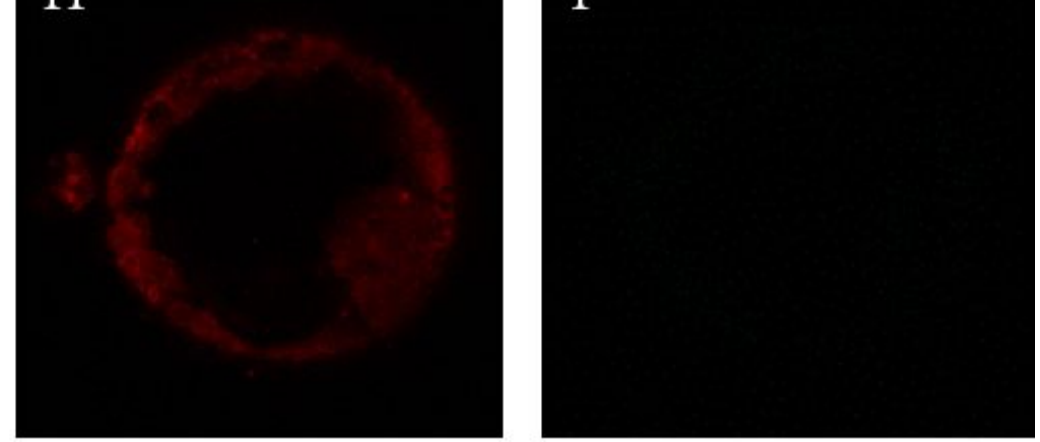

Figure 1

Representative image of immunofluorescence staining of ACE2 and NRP1 in human oocytes $(a-c, n=8)$, cleavage embryos ( $d-f, n=10)$ and blastocysts ( $\mathrm{g}-\mathrm{i}, \mathrm{n}=6$ ). Co-staining for NRP1 (green) and ACE2 (red) showed presence of ACE2 at the membrane and cytoplasm of TE cells and ICM. The fluorescence signal of ACE2 is the strongest in blastocyst stage (h), much weaker in cleavage embryos (e) and weakest in GV oocytes (b). NRP1 (green fluorescence signal) is barely visible in any detected stages (c, $\mathrm{f}, \mathrm{i})$. Nuclei were stained with Hoechst 33342 (blue) (a, d, g). Scale bar, $20 \mu \mathrm{m}$. 


\section{Hoechst}
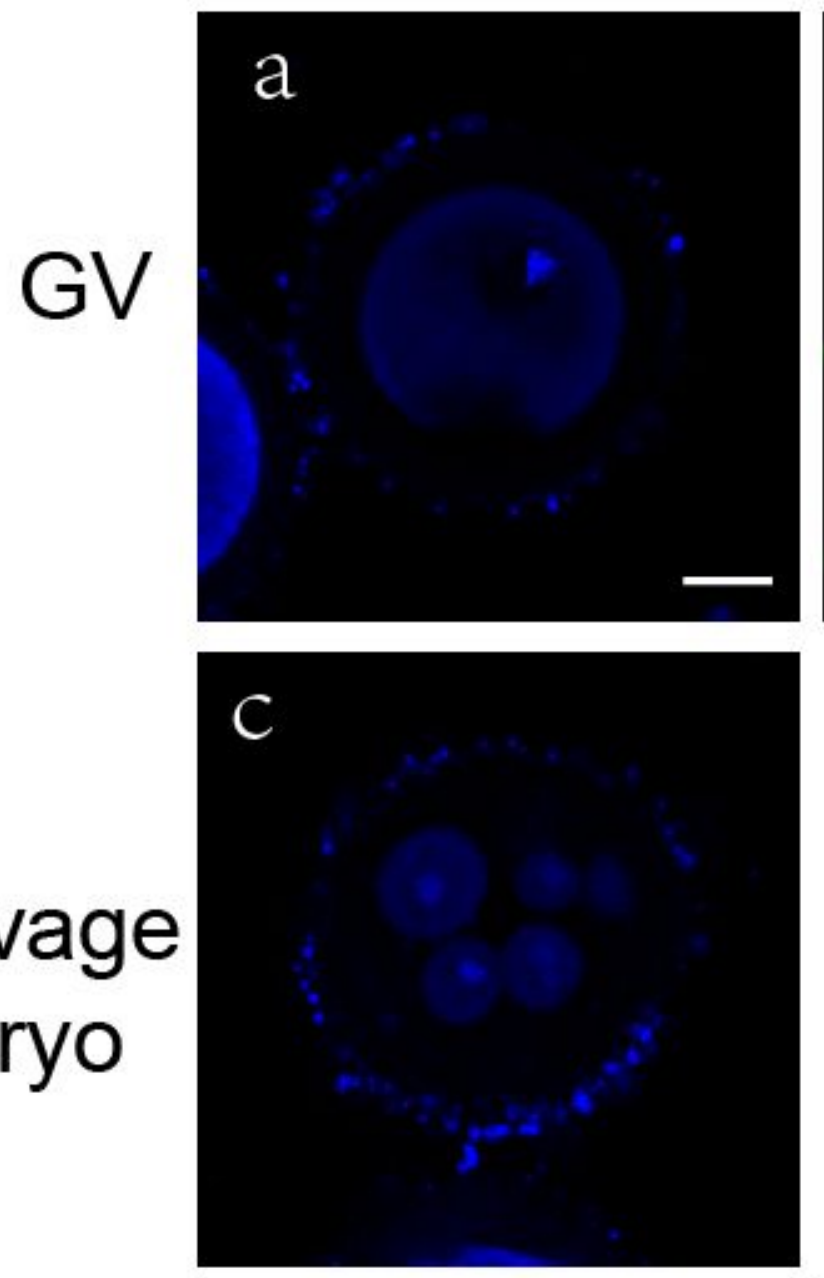

Cleavage embryo

\section{TMPRSS2}
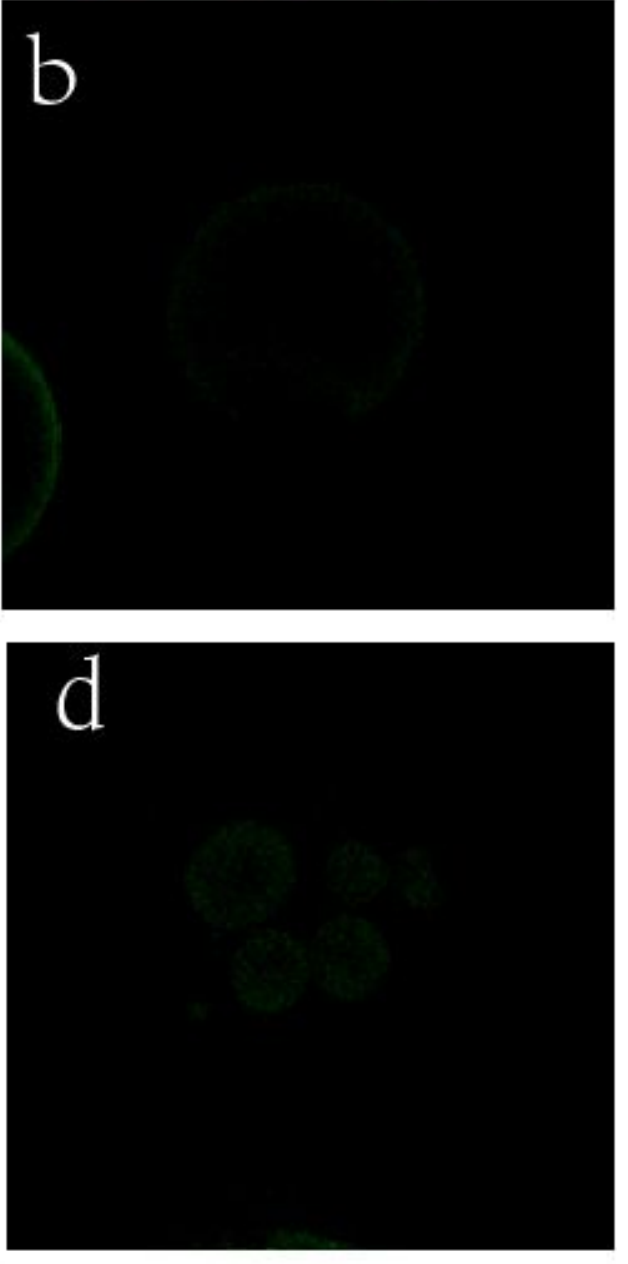

e

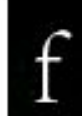

\section{Blastocyst}

\section{Figure 2}

Representative image of immunofluorescence staining for TMPRSS2. The result showed that TMPRSS2 signal was mainly present in TE $(n=5)$. Nuclei were stained with Hoechst 33342 (blue). Scale bar, $20 \mu \mathrm{m}$. 
The expression of ACE2 and TMPRSS2 in human oocytes and preimplantation embryos

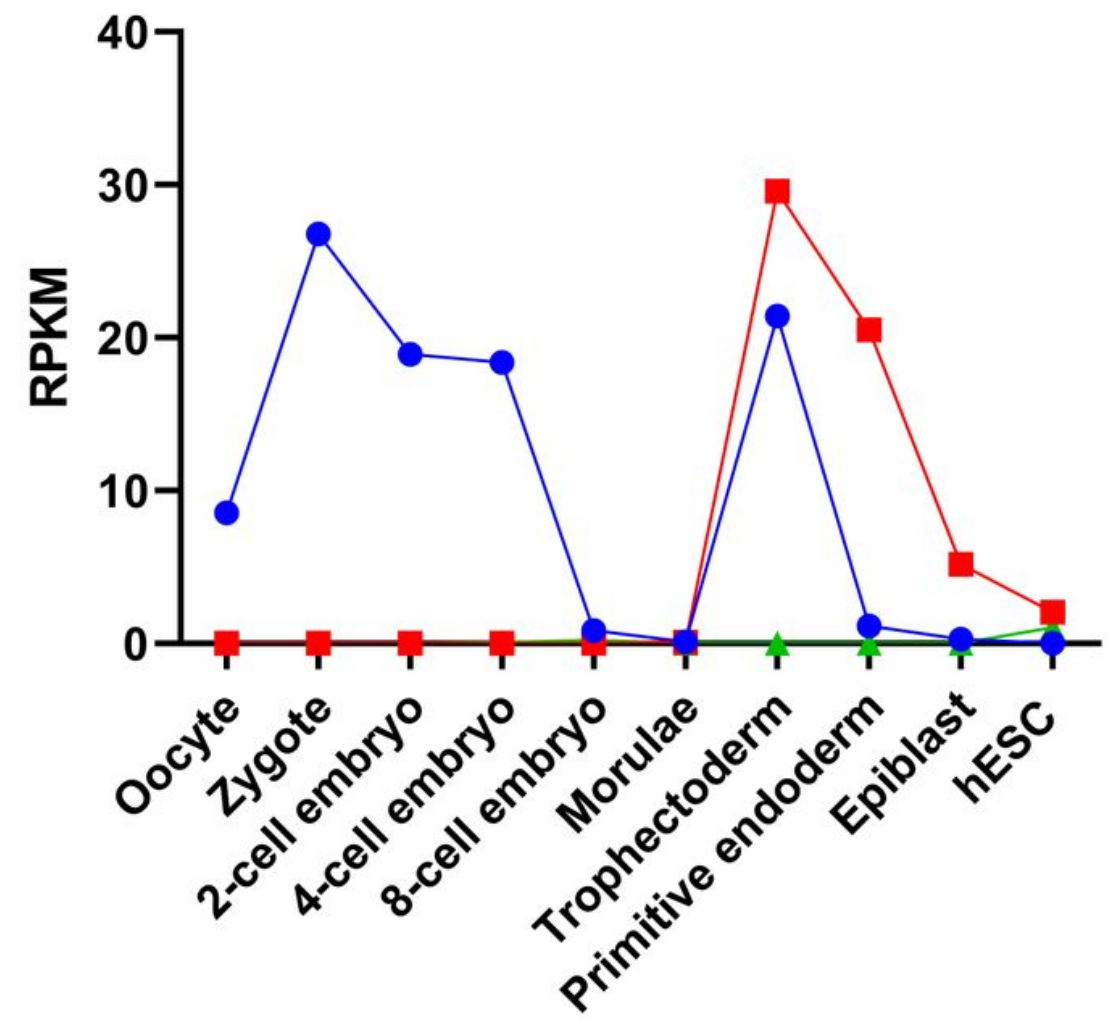

$\rightarrow$ ACE2

- TMPRSS2

$\leftarrow$ NRP1

Figure 3

Expression patterns of ACE2, TMPRSS2 and NRP1 in human oocytes and preimplantation embryos from transcriptome examination.

\section{Supplementary Files}

This is a list of supplementary files associated with this preprint. Click to download.

- FigS1.jpg 